\title{
Diplopía y ptosis palpebral izquierda como primera manifestación de metástasis hipofisarias de adenocarcinoma pulmonar
}

\section{Diplopia and left palpebral ptosis as a first manifestation of hypophysary metastases of pulmonary adenocarcinoma}

\author{
S. Junquera ${ }^{1}$, J. Verdún ${ }^{2}$, M.C. Bacaicoa ${ }^{3}$, M. Mendigaña ${ }^{3}$, J. Lafita ${ }^{1}$
}

\section{RESUMEN}

Paciente sin antecedentes patológicos de interés que debuta con diplopía y ptosis palpebral izquierda como primera manifestación de metástasis hipofisarias de un cáncer de origen pulmonar ampliamente diseminado y no conocido previamente. El diagnóstico se hizo con pruebas de imagen específicas del área hipofisaria en donde se apreció una neoformación en silla turca, que invadía el seno cavernoso izquierdo con erosión del dorso selar, junto con la presencia de nódulos pulmonares y múltiples adenopatías, además del estudio histológico de las lesiones. Se detectó un hipogonadismo hipogonadotropo como única alteración hormonal debido a la infiltración de la hipófisis anterior por las metástasis óseas en la base del cráneo. El paciente, a pesar de recibir varias sesiones de radioterapia, sufrió un deterioro neurológico progresivo falleciendo a las pocas semanas.

Palabras clave. Metástasis hipofisarias. Hipogonadismo hipogonadotropo. Diplopía. Cáncer pulmonar.

\begin{abstract}
A patient, without significant previous medical history, who started with diplopia and left palpebral ptosis as the first manifestation of hypophysary metastases of a widely disseminated and previously undetected cancer of pulmonary origin. Together with a histological study of the lesions, the diagnosis was made with specific image tests of the hypophysary area, which detected a neoformation in the sella turca that was invading the left cavernous sinus with erosion of the verso of the sellar, together with the presence of pulmonary nodules and numerous adenopathies. An hypogonadotropic hypogonadism was detected as the only hormonal alteration due to the infiltration of the anterior hypophysis due to the osseous metastases at the base of the cranium. The patient, in spite of receiving several weeks of radiotherapy, suffered a progressive neurological deterioration and died after a few weeks.
\end{abstract}

Key words. Hypophysary metastases. Hypogonadotropic hypogonadism. Diplopia. Lung cancer.
1. Servicio de Endocrinología. Complejo Hospitalario de Navarra. Pamplona.

2. Servicio de Oncología. Complejo Hospitalario de Navarra. Pamplona.

3. Servicio de Radiología. Complejo Hospitalario de Navarra. Pamplona.

Recepción: 28 de septiembre de 2011

Aceptación provisional: 9 de noviembre de 2011

Aceptación definitiva: 19 de diciembre de 2011

\section{Correspondencia:}

Sonia Junquera Bañares

Complejo Hospitalario de Navarra A

C/ Irunlarrea, 3

31008 Pamplona

Tfno. 848422038

E-mail: sojuba@hotmail.com 


\section{INTRODUCCIÓN}

En el 5\% de los pacientes oncológicos se detectan metástasis hipofisarias en el contexto de una diseminación tumoral importante, y sólo en un $7 \%$ son sintomáticas. En el $56 \%$ de los pacientes los síntomas neurológicos y/o alteraciones hormonales con los que debutan, son la primera manifestación de un cáncer oculto ${ }^{1}$, y en un $3 \%$ de los casos, el tumor primario no se detecta a pesar de una investigación exhaustiva ${ }^{2}$.

Las metástasis hipofisarias son más frecuentes en pacientes con cáncer de mama y pulmón. En su debut predominan los síntomas neurológicos, y las alteraciones hormonales asociadas que se detectan con más frecuencia son las derivadas de la infiltración de la neurohipófisis ${ }^{3}$.

\section{CASO CLÍNICO}

Varón de 64 años que acude al servicio de Urgencias por presentar, desde hacía 3 días, debilidad generalizada, sensación de inestabilidad, sin giro de objetos, cefalea hemicraneal izquierda, con disminución de la apertura palpebral izquierda y diplopía binocular.

Antecedentes personales: Nefrolitiasis bilateral que requirió litotricia extracorpórea en varias ocasiones. Morfea en placas. Exfumador de 1 paquete/día desde hacía 12 años. Exploración: Exploración general sin nada reseñable. Exploración neurológica: ptosis palpebral izquierda, paresia de recto externo izquierdo, alteración de la estática, con tendencia a la caída hacia la derecha y dismetría dedo-nariz izquierda. Analítica y TAC craneal normales.

Ingresa en Neurología para estudio con sospecha de ACV isquémico de territorio vertebrobasilar, presentando a las 24 horas del ingreso afectación pupilar.

Pruebas complementarias de laboratorio: hemograma y bioquímica general normales, salvo el fósforo inorgánico: $5,1 \mathrm{mg} / \mathrm{dL}(2,7-4,5)$ y LDH: $556 \mathrm{U} / \mathrm{L}$ (240-480). Estudio hormonal: TSH: $0,432 \mathrm{mU} / \mathrm{L}(0,4-4)$; T4 libre: $0,97 \mathrm{ng} / \mathrm{dL}(0,7-1,42)$; FSH: 3,87 UI/L (0,7-11,1); LH: 1,65 UI/L $(0,87-7,6)$; testosterona libre: $2,3 \mathrm{ng} / \mathrm{L}$ (5,6-19); IGF-1: 231 $\mu \mathrm{g} / \mathrm{L}$ (75-212); cortisol plasmático: 6,96 $\mu \mathrm{g} / \mathrm{L}(5-$ 25); ACTH indetectable (los datos de cortisol y ACTH no son valorables por tratamiento con corticoides); PRL: 11,3 $\mu \mathrm{g} / \mathrm{L}$ (2,1-13); GH: 0,92 $\mu \mathrm{g} / \mathrm{L}(0,06-5)$; ADH: $3,2 \mathrm{pg} / \mathrm{mL}$ (hasta 7,6); Osmolalidad en sangre: $285 \mathrm{mOs} \mathrm{m} / \mathrm{L}$; Osmolaridad en orina: $420 \mathrm{mOsm} / \mathrm{L}$. Marcadores tumorales: $\mathrm{Ca}$
19,9: 67,2 KU/L (0-33); CYFRA 21,1: 76,87 (0-3,3) y enolasa neuronal específica: $22,89 \mu \mathrm{g} / \mathrm{L}(0-12,5)$.

Estudio de imagen: la ARM descarta la presencia de aneurisma. En resonancia magnética craneal se aprecia una neoformación en silla turca, desplazada lateralmente hacia la izquierda, que invade el seno cavernoso ipsilateral, con un diámetro trasverso de $22 \mathrm{~mm}$ y anteroposterior de $12 \mathrm{~mm}$. Se realiza TAC de la silla turca para valorar afectación ósea, apreciando erosión del dorso selar, que plantea el diagnóstico diferencial entre metástasis en base de cráneo y cordoma de clivus (Fig. 1, 2). En TAC toracoabdominal se detecta un nódulo pulmonar espiculado, en LSI, de 22 x $20 \mathrm{~mm}$, con adenopatías mediastínicas e hiliares ipsilaterales y dos nódulos menores de $1 \mathrm{~cm}$ en hemitórax contralateral.

Estudio anatomopatológico: se hizo una biopsia de la masa hipofisaria, por vía transesfenoidal, una citología con aguja fina del nódulo pulmonar y otra de un nódulo en músculo paraespinal izquierdo con diagnóstico AP de carcinoma poco diferenciado de probable extirpe pulmonar en las tres muestras.

Evolución: durante el estudio, el cuadro neurológico se fue deteriorando, apareciendo ptosis palpebral bilateral, por afectación del III par bilateral, con pupilas midriáticas y arreactivas. Dada la situación clínica se realizó radioterapia paliativa de urgencia, sobre la lesión de la base de cráneo, hasta un total de 30 Gy (fraccionada durante 10 días), con mejoría parcial de la cefalea y de la parálisis del III par bilateral. Progresivamente el paciente presentó dolor intenso a nivel cervical y lumbosacro, confirmando en la gammagrafía ósea la presencia de áreas de hipofijación en D11 y L4, al igual que al realizar PET, como estudio de extensión, que confirmó la presencia de depósitos hipermetabólicos en LSI pulmonar, hilio pulmonar izquierdo, ventana aortopulmonar, con importante afectación esquelética, más acusada en: últimas vértebras cervicales y partes blandas adyacentes, pedículo derecho de D11, esternón, huesos pélvicos, cadera izquierda e implantes musculares en músculos pterigoides externos bilaterales, músculo constrictor inferior de la faringe, músculos dorsales largos de la espalda, pared abdominal, músculo intercostal derecho y en ambas extremidades inferiores.

Se realizó radioterapia antiálgica durante 15 días, a nivel de columna cervical y lumbosacra, con alivio parcial del dolor, y se dio de alta con tratamiento sintomático. Diez días después, el paciente acude al servicio de Urgencias con un cuadro de deterioro severo, falleciendo a las pocas horas. 

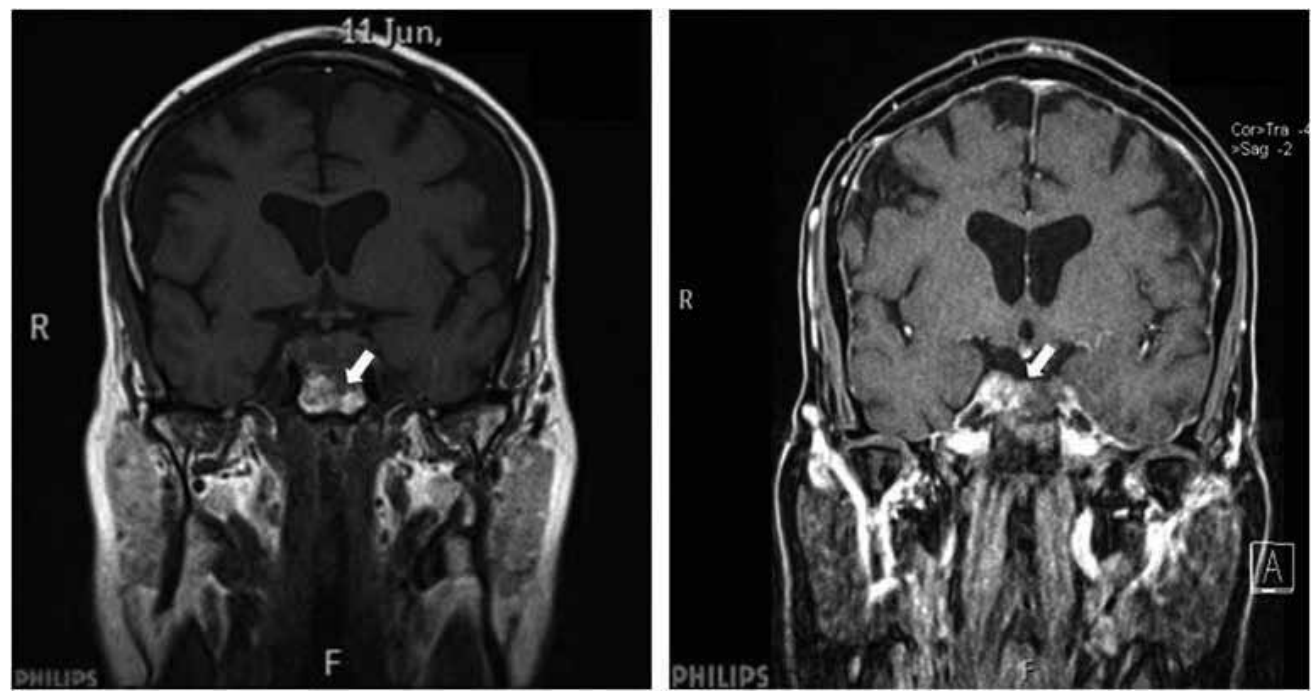

Figura 1. Izda. T1 coronal sin contraste. Dcha. T1 coronal con gadolinio.

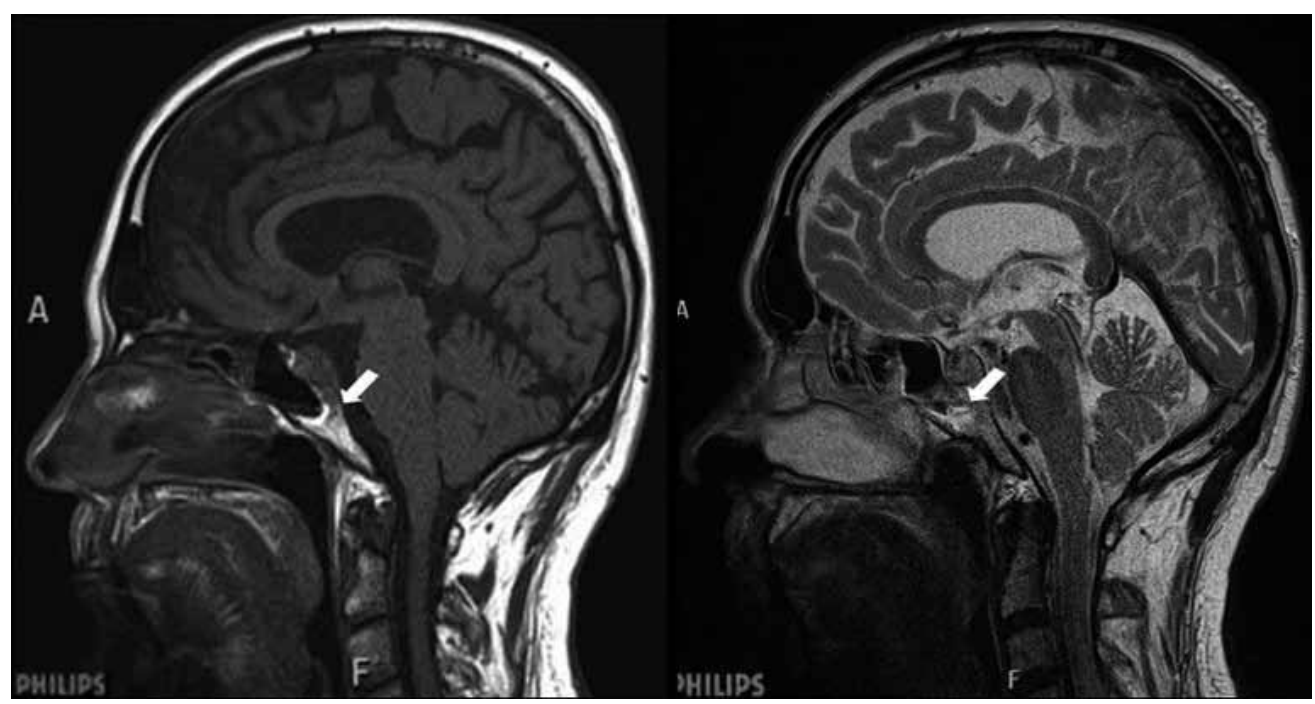

Figura 2. Izda-T1 sagital sin contraste. Dcha-T2 sagital.

\section{DISCUSIÓN}

El primer caso de metástasis hipofisarias fue descrito en 1857 por L. Benjamín a raíz de una autopsia practicada a un paciente con melanoma diseminado.

El 1\% de las masas hipofisarias vistas en series de cirugía transesfenoidal son metástasis hipofisarias. Sin embargo, la prevalencia aumenta al $3,6-5 \%$ en autop- sias de pacientes con tumores malignos, y hasta un $17,6-29 \%$ en el caso de mujeres fallecidas por carcinoma de mama diseminado. Los cánceres que con más frecuencia metastatizan a hipófisis son el de mama en la mujer y el de pulmón en el hombre, abarcando entre ambos dos tercios del total.

El diagnóstico de metástasis hipofisarias está aumentando debido a la mayor 
sensibilidad de las técnicas de radiodiagnóstico y a la mayor supervivencia de los pacientes oncológicos. Es más frecuente su diagnóstico en la sexta o séptima década de la vida, en cánceres diseminados con metástasis en cinco o más localizaciones y preferentemente óseas ${ }^{3}$.

Los síntomas neurológicos más frecuentes con los que debutan las metástasis hipofisarias son: cefalea, alteración del campo visual con hemianopsia bitemporal por compresión del quiasma óptico, ptosis palpebral y oftalmoplejia. Es común que se den déficits hormonales asociados, por la infiltración de la hipófisis y estructuras adyacentes por las células tumorales.

La hipófisis es una glándula con gran capacidad de reserva y para que se detecten déficits hormonales necesita estar infiltrada en un $75-80 \%$. Puede verse afectada la neurohipófisis, adenohipófisis o hipotálamo, y generalmente, los síntomas se detectan por la aparición de alteraciones hormonales. Sólo hemos encontrado un caso de metástasis hipofisarias de cáncer folicular de tiroides que debutó con síntomas neurológicos sin la presencia de déficits hormonales asociados ${ }^{4}$.

En el paciente que presentamos se detectó al diagnóstico como única alteración hormonal un hipogonadismo hipogonadotropo, que no fue el motivo de consulta. Es lógico que las células gonadotropas sean las primeras en afectarse en caso de infiltración tumoral por su escaso número en proporción a los otros tipos celulares y por su situación en la periferia de la hipófisis. Generalmente es más frecuente la afectación de neurohipófisis, especialmente en tumores hematológicos y pulmonares. Sin embargo, se ha visto que el cáncer de mama tiene especial predilección por la adenohipófisis y esto parece explicarse porque este medio rico en prolactina podría favorecer la proliferación de esta extirpe celular ${ }^{5}$.

La alteración más frecuente en las metástasis hipofisarias es la diabetes insípida $(29-71 \%)^{6}$. De hecho, se estima que el $20 \%$ de los casos de diabetes insípida del adulto se deben a metástasis hipofisarias. Se ha visto que es la única deficiencia que puede revertir después de tratar el tumor y parece ser por la existencia de un mecanismo de regeneración axonal. Está descrito un caso de metástasis hipofisarias por cáncer de esófago que debutó con diabetes insípida y panhipopituitarismo, y que posteriormente, se normalizó la diuresis al disminuir la masa hipofisaria con quimioterapia, pero el resto de deficiencias hormonales no mejoraron?.

Son raros los casos en los que se detecta hiperproducción hormonal como consecuencia de metástasis hipofisarias. Hay descrito un caso de tumor endocrino productor de GHRH que metastatiza a la glándula pituitaria y causa localmente hiperestimulación con hiperplasia somatotrofa $\mathrm{y}$ transformación adenomatosa ${ }^{8}$. Es poco frecuente en las metástasis hipofisarias que se dé un aumento de prolactina (6,3\%), y generalmente se detecta en el caso de metástasis hipotalámicas donde suele estar infiltrado el infundíbulo y se inhibe la llegada de dopamina, y con ello, se para la inhibición que ésta produce sobre la secreción de PRL. Sin embargo, es más frecuente la presencia de panhipopituitarismo en este tipo de lesiones por la interrupción de factores liberadores que estimulan la producción de hormonas adenohipofisarias ${ }^{9,10}$.

La mayor incidencia de metástasis en el lóbulo posterior se debe al aporte sanguíneo procedente directamente de las arterias hipofisarias, a la circulación sistémica y a poseer un área de contacto mayor con la dura adyacente. Sin embargo, el lóbulo anterior lo irriga el sistema venoso portal, y secundariamente, el sistema infundibular inferior que procede del lóbulo posterior. Por ello, las metástasis del lóbulo anterior suelen ser el resultado de la diseminación contigua del lóbulo posterior. El lóbulo anterior parece ser más susceptible a infartos isquémicos y se suele asociar con lesiones metastásicas de mayor tamaño que las de la hipófisis posterior ${ }^{3}$.

Aunque es menos frecuente, hay descritos casos similares al nuestro, en los que se afecta exclusivamente el lóbulo anterior por infiltración a través de la pared ósea de la silla turca y no por diseminación hematógena. En nuestro paciente puede ser que 
las metástasis óseas en la base del cráneo detectadas en el TAC, sean las responsables de la afectación de la hipófisis anterior por contigüidad. Es difícil diferenciar metástasis hipofisarias de metástasis óseas en la base del cráneo, pero al parecer el aumento de esclerosis que se detecta en éstas, es punto clave para ello. Existe un caso descrito además del que exponemos en el que se observan metástasis hipofisarias de cáncer pulmonar exclusivamente en adenohipófisis y en los dos pacientes coincide tener metástasis óseas cervicales que pudieran haber metastatizado a la base del cráneo ${ }^{11}$.También se ha observado este fenómeno en cáncer de hígado, riñón y tiroides, presumiblemente por el mecanismo descrito ${ }^{3,12}$.

En el estudio de toda masa hipofisaria debe plantearse el diagnóstico diferencial de metástasis hipofisarias, especialmente en los pacientes mayores de 55 años con diabetes insípida central o con panhipopituitarismo de rápida instauración y síndrome constitucional de etiología no filiada.

Hay signos radiológicos que ayudan al diagnóstico de malignidad: crecimiento rápido de una masa selar y paraselar, erosión del suelo selar, pérdida de señal en T1 de neurohipófisis, ensanchamiento del tallo hipofisario, invasión del seno cavernoso o esclerosis de la silla turca. La RMN de hipófisis tiene mayor rentabilidad que el TAC craneal en estos casos, puesto que éste puede ser normal como fue en nuestro paciente. Sólo tendría utilidad la realización de TAC de silla turca en el caso que sospecháramos metástasis óseas en la base del cráneo.

La evolución del caso que presentamos es muy rápida, especialmente por la agresividad del tumor, con supervivencia de 50 días entre el primer síntoma y el fallecimiento. El hallazgo de metástasis en hipófisis indica mal pronóstico a corto plazo y se estima una supervivencia media de 6 a 22 meses $^{1}$. El tratamiento de QT/RT/cirugía descompresiva suele ser paliativo para mejorar los síntomas neurológicos, pero no interfiere con el avance de la enfermedad ni tampoco se ha demostrado que mejore la supervivencia.

\section{BIBLIOGRAFÍA}

1. Morita A, Meyer FB, Laws ER. Symptomatic pituitary metastases. J Neurosurg 1998; 89: 69-73.

2. Frago Marquínez I, Fuentes Gómez C, Maldonado Castro M, Puelles Emaldibarra N, San Miguel López de UrRalde S, Echeveste Aizpurua J et al. Metástasis hipofisarias en pacientes con antecedentes neoplásicos. Endocrinol Nutr 2009; 56: 265-269.

3. Komninos J, Vlassopoulou V, Protopapa D, Korfias S, Kontogeorgos G, SAKas DE et al. Tumors metastatic to the pituitary gland: case report and literature review. J Clin Endocrinol Metab 2004; 89: 574-580.

4. Prodam F, Pagano L, Belcastro S, Golisano G, Busti A, SAMÁ M et al. Pituitary metastases from follicular thyroid carcinoma. Thyroid 2010; 20: 823-830.

5. Azambuja E, Piccart MJ, Awada A. Long-term survival in pituitary metastasis from breast cancer. Breast 2006; 15: 446-447.

6. FASSET DR, Couldwell WT. Metastases to the pituitary gland. Neurosurg Focus 2004; 16: E8.

7. López-MondéJar P, Massuti B, Pardo C, Moreno O, Aznar S, Picó A. Panhipopituitarismo secundario a metástasis hipofisaria de adenocarcinoma esofágico. Endocrinol Nutr 2004; 51: 133-135.

8. Nasr C, Mason A, Mayberg M, Staugaitis SM, Asa SL. Acromegaly and somatotroph hyperplasia with adenomatous transformation due to pituitary metastasis of a growth hormonereleasing hormone-secreting pulmonary endocrine carcinoma. J Clin Endocrinol Metab 2006; 91: 4776-4780.

9. Ko JC, Yang PC, Huang TS, Yeh KH, Kuo SH, LuH KT. Panhypopituitarism caused by solitary parasellar metastasis from lung cancer. Chest 1994; 105: 951-953.

10. Guerra Alía E, Garrido López P, Pachón Olmos V, Medina Martínez J, González Martín A, Val SAlgado MA. Hipopituitarismo secundario debido a metástasis en región hipotalámica de carcinoma microcítico de pulmón. Endocrinol Nutr 2009; 56: 201-204.

11. Ito I, Ishida T, Hashimoto T, Arita M, Osawa M, YокотA $\mathrm{T}$ et al. Hypopituitarism due to pituitary metastasis of lung cancer: case of a 21-year-old man. Intern Med 2001; 40: 414-417.

12. Kramer CK, Ferreira M, Silveiro SP, Gross JL, Dora JM, Azevedo MJ. Pituitary gland metastasis from renal cell carcinoma presented as a non-functioning macroadenoma. Arq Bras Endocrinol Metabol 2010; 54: 498-501. 
